\title{
Preface
}

\section{Where Are the Advancements in the Management of Mandibular Condylar Process Fractures?}

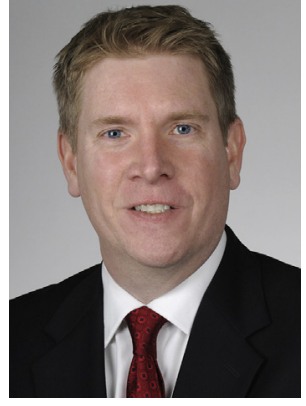

Martin B. Steed, DDS, FACS Editor
Many surgical lectures begin with an introduction describing the history of the management of an injury or pathology. This is then followed by a sequential discussion of the sentinel modifications that have been witnessed and accepted. When one listens to such a talk on mandibular condyle fractures, it is sometimes apparent that, for many of these injuries, we still treat them in a manner similar to the first slide on the PowerPoint.

But while the debate on optimal treatment of fractures of the mandibular condylar process continues, it does so now in an environment in which we are witnessing accelerated advancement in surgical technology. Improved imaging, instrumentation, fixation methods/materials, and virtual surgical planning provide us with novel opportunities to improve care for our patients. Our clinical outcomes research is beginning to show a trend toward improved outcomes with open treatment.

It was within this environment that I was recently fortunate enough to be a part of a Strasburg Osteosynthesis Research Group advanced module dedicated exclusively to the management of mandibular condylar process fractures. Listening to presentations from the likes of Ed Ellis, Richard Loukota, Eric Dierks, David Powers, and Stephen Macleod, I realized that advances are, in fact, being made. We are now beginning to understand what the adaptations are that allow most patients to accommodate and retain function after a condylar process fracture. We have become better able to classify these injuries (especially intracapsular/dicapitular) and anticipate those that may not be amenable to closed treatment and subsequent adaptations. We are getting better at identifying the limitations of closed treatment.

For those fractures that have classically been more difficult to treat, such as high severely displaced fractures, we now have been introduced to approaches, instrumentation, and fixation methods that were not previously within our armamentarium. Virtual surgical planning and intraoperative navigation are actively being explored for the trauma patient.

I felt that the illustrative nature of this Atlas of the Oral and Maxillofacial Surgery Clinics of North America would lend itself well to clearly showing facial trauma surgeons where these advances are being made.

Martin B. Steed, DDS, FACS Medical University of South Carolina Department of Oral and Maxillofacial Surgery

173 Ashley Avenue

BSB 453 MSC 507

Charleston, SC 29425, USA

E-mail address: steedma@musc.edu 